\title{
Polibotánica
}

ISSN electrónico: 2395-9525

POLIBETÁNICA polibotanica@gmail.com Instituto Politécnico Nacional México http:www.polibotanica.mx

\section{DIVERSIDAD DE HELECHOS Y LICOPODIOS EN EL ÁREA PRIVADA DE CONSERVACIÓN TALHPAN, PAPANTLA, VERACRUZ, MÉXICO}

\section{DIVERSITY OF FERNS AND LYCOPODS IN THE TALHPAN PRIVATE CONSERVATION AREA, PAPANTLA, VERACRUZ, MEXICO}

Barrios-Morales, F.; J.L. Alanís-Méndez, y C.I. Carvajal-Hernández.

DIVERSIDAD DE HELECHOS Y LICOPODIOS EN EL ÁREA PRIVADA DE CONSERVACIÓN TALHPAN, PAPANTLA, VERACRUZ, MÉXICO.

DIVERSITY OF FERNS AND LYCOPODS IN THE TALHPAN PRIVATE CONSERVATION AREA, PAPANTLA, VERACRUZ, MEXICO.

POLIBETÁNICA

Instituto Politécnico Nacional
Núm. 47: 1-12 México. Enero 2019

DOI: $10.18387 /$ polibotanica.47.1 


\section{DIVERSIDAD DE HELECHOS Y LICOPODIOS EN EL ÁREA PRIVADA DE CONSERVACIÓN TALHPAN, PAPANTLA, VERACRUZ, MÉXICO}

\section{DIVERSITY OF FERNS AND LYCOPODS IN THE TALHPAN PRIVATE CONSERVATION AREA, PAPANTLA, VERACRUZ, MEXICO}

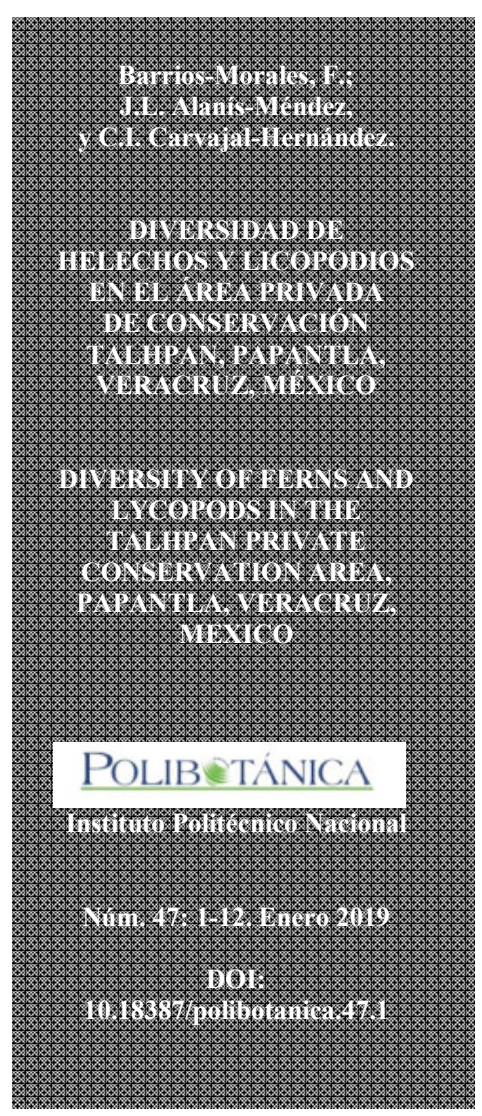

\author{
F. Barrios-Morales \\ J.L. Alanís-Méndez \\ Facultad de ciencias Biológicas y Agropecuarias. Universidad Veracruzana. \\ Carretera Tuxpan-Tampico Km. 7.5 Col. Universitaria, \\ CP 92860, Tuxpan, Veracruz, México.
}

C.I. Carvajal-Hernández / ccarvajal@uv.mx Instituto de Investigaciones Biológicas. Universidad Veracruzana. Av. Dr. Luis Castelazo Ayala s/n Col. Industrial Ánimas, CP 91190, Xalapa, Veracruz, México.

RESUMEN: El conocimiento de la pteridoflora de la zona norte de Veracruz es escaso, y más aún cuando se habla de la riqueza de helechos y licopodios en las zonas de baja altitud. El objetivo del estudio fue analizar la diversidad de helechos y licopodios en un fragmento de selva mediana subperennifolia. Se establecieron 15 parcelas de $20 \times 20 \mathrm{~m}$, en tres hábitats (fragmento de selva, vegetación ribereña y cultivo de cítricos), para analizar la riqueza, abundancia y recambio de especies de estos grupos en distintos hábitats. Además, se determinaron las especies presentes en zonas aledañas a la reserva. Se registró un total de 22 especies de helechos y licopodios; 17 de ellas estaban presentes en las parcelas de muestreo y el resto se encontraron en sitios aledaños. El hábitat ribereño presentó la mayor riqueza específica, seguido del fragmento de selva; estos dos hábitats tuvieron una similitud florística de $22 \%$. La zona de cultivo presentó la menor riqueza específica. La selva representa un refugio importante para la diversidad de helechos, sin embargo, la vegetación ribereña fue la que mayor diversidad presentó. El recambio de especies fue alto entre ambientes, lo cual tiene un valor para los planes de conservación, ya que es necesario incluir la heterogeneidad paisajística en las propuestas de preservación de los ecosistemas.

Palabras clave: diversidad alfa y beta; inventario florístico; pteridofitas; selva mediana subperennifolia; vegetación ribereña.

ABSTRACT: The knowledge of the pteridophyta in flora of the northern zone of Veracruz is scarce, and ever more when it comes to species richness of ferns and lycopods in the elevational low zones. The aim of this research is to analyze the diversity of ferns and lycopods in a semideciduos forest. Were established 15 plots of $20 \times 20 \mathrm{~m}$ in three habitats (semideciduos forest fragment, riparian vegetation and citrus cultivation) to analyze the richness, abundance and turnover of species of these groups in different habitats. In addition, the species present in areas bordering the reserve were registered. A total of 22 species of ferns and lycopods were recorded, of these, 17 correspond to the sampling plots and the rest were found in adjacent sites. The riparian habitat presented the highest specific richness, followed by the semideciduos forest fragment; these two habitats had a floristic similarity of $22 \%$. Citrus cultivation had the lowest specific richness. The forest in the research area represents a refuge for the diversity of ferns, however the riparian vegetation was the one that presented the greatest diversity. The replacement of species in the different 
habitats is high, which has a value for conservation plans, since it is necessary to include landscape heterogeneity in the proposals for the preservation of ecosystems.

Key words: alpha and beta diversity; floristic inventory; pteridophyte; riparian vegetation; semideciduos forest.

\section{INTRODUCCIÓN}

La heterogeneidad paisajística, definida por la variedad de climas y la complejidad histórica, geológica y biológica, se ve reflejada en la presencia de una importante diversidad florística que distingue a México como un país megadiverso (Sarukhán et al., 1996; SEMARNAT, 2009). Se ha calculado que contiene 22969 especies (de las cuales cerca del 50\% son endémicas) lo que lo ubica como el tercer país con mayor riqueza de plantas en América Latina, después de Brasil (con alrededor de 33160 especies) y Colombia (23 100 especies) (Villaseñor, 2016; Ulloa et al., 2017; Villaseñor, 2016; Ulloa, y otros, 2017). Para el caso concreto de helechos y licopodios, se calcula que en México existen 1034 especies y a nivel mundial entre 11900 especies, lo que representa el 8.6\% de la pteridoflora mundial (Villaseñor, 2016; PPGI, 2016).

Veracruz es el estado que, después de Chiapas y Oaxaca, cuenta con el mayor número de plantas vasculares de México, con 8497 especies (Villaseñor, 2016), de las cuales 564 son helechos y licopodios (Krömer et al., 2015; Tejero-Díez et al., 2011; Carvajal-Hernández, Guzmán-Jacob, Smith, \& Krömer, 2018). Además, es una de las regiones que cuenta con mayor diversidad de tipos de vegetación en el país (Castillo-Campos et al., 2011). Esto es debido a un amplio intervalo altitudinal desde el nivel del mar hasta más de $5000 \mathrm{~m}$, lo que permite la presencia de zonas frías, templadas y cálidas, así como climas que van desde húmedos a subhúmedos (Carvajal-Hernández et al., 2017; Gómez-Pompa et al., 2010). Sin embargo, actualmente por las actividades antropogénicas, la vegetación original del estado se ha reducido en más del $15 \%$ y los tipos de vegetación se encuentran en su mayoría concentrados en fragmentos aislados (Castillo-Campos et al., 2011).

Los helechos y licopodios son dos grupos taxonómicos de gran importancia para la biodiversidad de los ecosistemas tropicales, ya que representan entre el $13 \%$ al $22 \%$ de las floras locales, en particular en zonas montañosas (Kelly et al., 1994; Kessler, 2010). Son grupos cosmopolitas que se encuentran principalmente en zonas de media montaña, donde la humedad y temperatura templada son favorables para su supervivencia. No obstante, en las zonas de mayor altitud (por arriba de $2600 \mathrm{~m}$ ) y en las zonas bajas (a menos de $500 \mathrm{~m}$ ), la diversidad de especies decrece hasta un 90\% (Carvajal-Hernández \& Krömer, 2015; Kessler, 2001; Salazar et al., 2015). Una gran cantidad de especies de helechos son sensibles a los cambios en el microclima que provoca el disturbio antropogénico: por ejemplo el aumento de la temperatura y la disminución de la humedad del bosque (Barradas Paciencia \& Prado, 2005; CarvajalHernández, 2014; 2017), lo que es alarmante por la abrupta reducción de la vegetación original.

La mayoría de las investigaciones en Veracruz con ambos grupos de plantas se han realizado en la zona centro y sur (región de la reserva de la Biosfera de Los Tuxtlas) (Tejero-Díez et al., 2011) y en las zonas montañosas, pero se conoce en menor medida la pteridoflora que se encuentra en la región norte del estado y de manera general en las zonas de baja altitud de México (Acebey et al., 2015; Carvajal-Hernández \& Krömer, 2015; Cetzal-Ix et al., 2013). Además, se sabe poco sobre el recambio de especies de helechos en diferentes hábitats, y más aún si se trata de las zonas de selvas tropicales de tierras bajas (Carvajal-Hernández et al., 2017). Ante la escasez de estudios de helechos en la zona norte de Veracruz, se considera importante documentar la diversidad presente en esa región del estado. Además, el área de conservación privada Talhpan, es un sitio de interés biológico por representar un fragmento aislado de selva mediana subperennifolia con poca perturbación y protegido por las comunidades indígenas locales, de la etnia totonaca (Basáñez, Alanís, \& Badillo, 2008; Ticante, 2015). Debido a lo anterior, el objetivo de la presente investigación fue analizar la diversidad de 
las especies de helechos y licopodios en el área privada de conservación Talhpan, en el municipio de Papantla, ubicado en el norte del estado de Veracruz y con ello contribuir al conocimiento sobre la dinámica del ensamble de especies de helechos en los ecosistemas tropicales de baja altitud.

\section{MÉTODOS}

\section{Área de estudio}

El área privada de conservación Talhpan se encuentra en el ejido "El Remolino", su tamaño es de 24 ha y está ubicada en la porción centro-sureste del municipio de Papantla, Veracruz (Vázquez-Torres et al., 2010) (fig. 1). El clima es cálido-subhúmedo con lluvias en verano, la temperatura media anual es de $28^{\circ} \mathrm{C}$ y la precipitación acumulada anual fluctúa entre 1100 y $1600 \mathrm{~mm}$; el promedio de humedad relativa es de 40\% (INEGI, 2009). La zona cuenta con pequeños arroyos y manantiales que fluyen a través de la reserva y desembocan en el río Tecolutla. El sustrato es cárstico, con abundantes rocas aflorantes y suelos someros; el relieve es irregular (Basáñez et al., 2008).

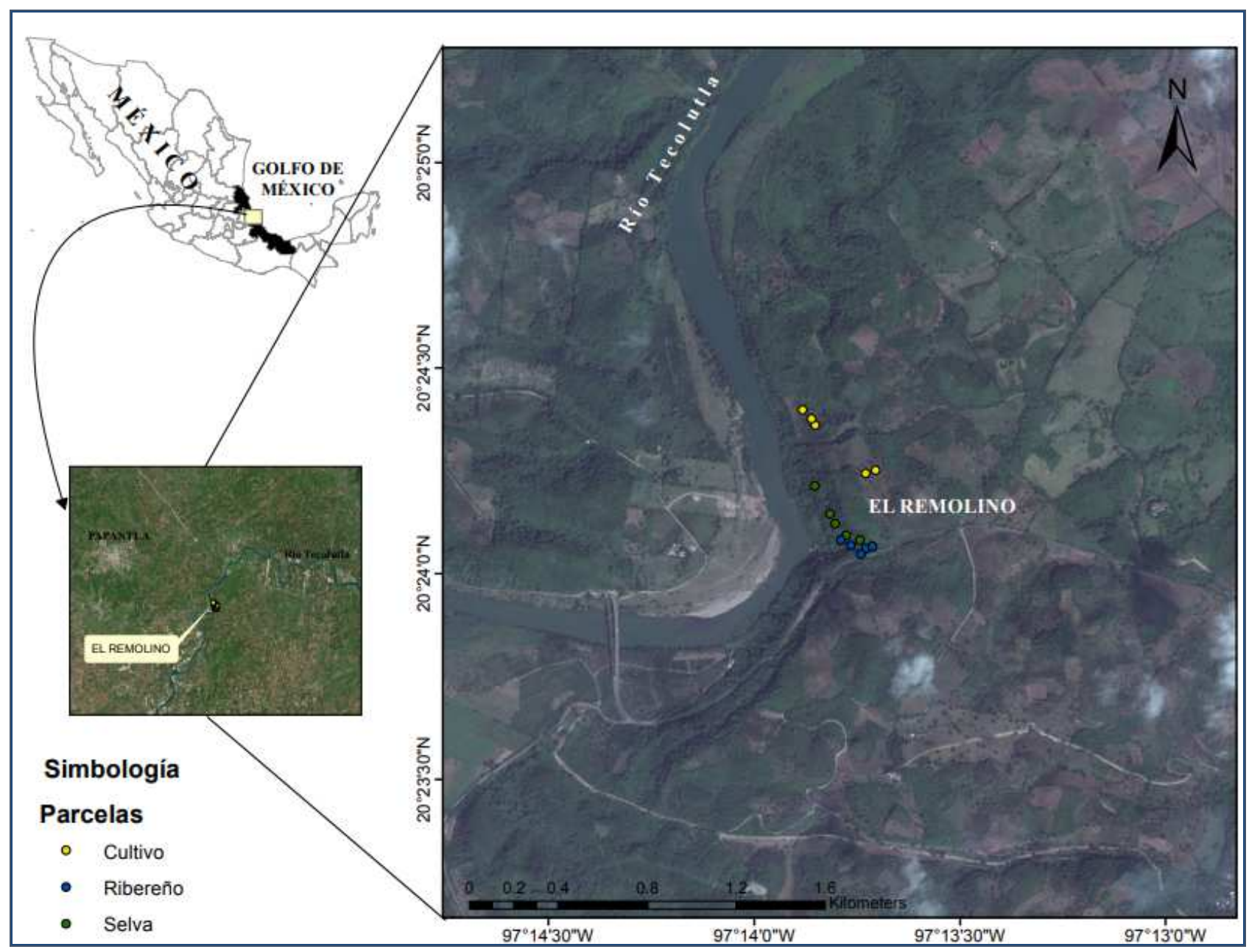

Fig. 1. Ubicación de la reserva privada de conservación Talhpan, en el ejido El Remolino, municipio de Papantla, Veracruz. 
La vegetación predominante en la zona de estudio es selva mediana subperennifolia, los árboles dominantes son: Aphananthe monoica (Hemsl.) J.-F. Leroy, Brosimum alicastrum Sw., Bursera simaruba (L.) Sarg., Carpodiptera ameliae Lundell, Cupania dentata DC., Dendropanax arboreus (L.) Decne. \& Planch., Ficus americana Aubl., Heliocarpus microcarpus Rose, Manilkara zapota (L.) P. Royen, Pimenta dioica (L.) Merr., Protium copal (Schltdl. \& Cham.) Engl. y Zuelania guidonia (Sw.) Britton \& Millsp. (Basáñez et al., 2008; Ticante, 2015). El sotobosque se encuentra representado por abundantes individuos del género Chamaedorea (Willd.), mientras las especies epifitas son escasas.

\section{Trabajo de campo}

Se seleccionaron tres microambientes para el muestreo, los cuales integran el mosaico paisajístico predominante en la zona: a) hábitat ribereño (RIB), formado por un arroyo en el interior de la selva que desemboca en el río Tecolutla; b) fragmento de selva mediana subperennifolia (SEL): compuesto por árboles de vegetación primaria (diámetros superiores a $15 \mathrm{~cm}$ ), con cobertura del dosel de más del $85 \%$ y poca representación de los estratos arbustivo y herbáceo en el sotobosque, la gente local indica que este sitio no ha sido sometido a disturbio; c) zona de cultivo (CUL): caracterizado por ser un monocultivo de cítricos sometido a deshierbe constante.

Se establecieron 15 parcelas de $20 \times 20 \mathrm{~m}$ (cinco por cada sitio de muestreo), donde se registró la presencia y abundancia de helechos y licopodios. (Kessler \& Bach, 1999). Además, se registraron las especies encontradas en dos ambientes anexos pertenecientes al área privada de conservación con la finalidad de complementar el inventario de helechos y licopodios. Estas zonas correspondieron en primer lugar a una comunidad vegetal conocida localmente como aguachal: humedal dominado por plantas herbáceas de aguas pantanosas o de agua dulce estancada. El segundo sitio corresponde a una zona de vegetación antropizada, la cual está representada por árboles remanentes de la selva, así como especies introducidas y utilizadas para diferentes fines. El muestreo en estos hábitats consistió en un recorrido, donde se registraron y colectaron los especímenes encontrados, pero con métodos de muestreo distintos por dos razones: 1) en la zona de aguachales, al estar siempre bajo la influencia del agua, no fue posible establecer parcelas de muestreo de 20 × 20 como en los otros sitios; 2) en la vegetación antropizada las personas introducen especies de helechos con fines alimenticios y/o medicinales, lo cual podría representar un error en el muestreo ya que no mostraría la dinámica natural del establecimiento de las especies. Los taxones registrados en esta zona fueron incluidos en el inventario florístico, pero no se consideraron en los análisis estadísticos, debido a las diferencias en el muestreo.

Las muestras colectadas fueron procesadas y depositadas en el herbario CIB, del Instituto de Investigaciones Biológicas de la Universidad Veracruzana en Xalapa, con duplicados depositados parcialmente en el Herbario Nacional de México (MEXU) del Instituto de Biología, UNAM. Los ejemplares se determinaron con las claves disponibles en Mickel y Smith (2004) y fueron comparados con los de herbario.

\section{Análisis estadístico}

El esfuerzo de muestreo de helechos y licopodios se evaluó con el estimador ACE, el cual se obtuvo mediante el programa EstimateS (Colwell, 2013). ACE (abundance-based coverage estimator) está basado en datos de abundancia y utiliza información adicional basada en aquellas especies con diez o menos individuos en la muestra (Moreno, 2001; Chao et al., 2005).

La diversidad de cada hábitat se evaluó utilizando el índice de Shannon-Wiener (índice de equidad), el cual asume que todas las especies están representadas en todas las muestras; indica además la uniformidad de las especies (en abundancia) teniendo en cuenta la totalidad del 
muestreo (Moreno, 2001). Para analizar la diversidad beta se evaluó el recambio de especies con el índice de Whittaker, el cual describe la diversidad beta como la variación en la composición de especies entre diferentes áreas (Koleff et al., 2003). La similitud florística entre las parcelas de muestreo se estimó con el análisis de agrupamiento y se representó en un dendrograma, con base en los valores de semejanza del índice de Jaccard. El índice y el dendrograma fueron calculados con el programa Past versión 2.17c (Hammer et al., 2001)

Para determinar si las diferencias numéricas relacionadas con la diversidad entre los hábitats estudiados eran o no significativas, se empleó el análisis de varianza (ANDEVA) de una vía. Adicionalmente, se realizó una comparación a posteriori, basada en una prueba pareada de Tukey (Programa Statistica v. 8.0) (StatSoft Inc., 2007), para conocer cuales sitios diferían entre sí.

Para determinar si existen especies asociadas a un tipo de hábitat en particular, se realizó el análisis IndVal, el cual es una expresión de la probabilidad de que una especie presente preferencias de hábitat, con base en la frecuencia y especificidad. Las especies con valor igual o superior a 0.5 son consideradas como indicadoras robustas para un sitio o tratamiento (Dufrene \& Legendre, 1997; Tejeda-Cruz et al., 2008). El análisis fue realizado con el paquete estadístico R, utilizando el paquete labdsv 1.5-0 (v 3.1.2; R Foundation for Statistical Computing, Vienna AT); Development Core team; versión 3.1.2).

\section{REsultados}

Diversidad alfa. Se registró un total de 22 especies de helechos y licopodios tanto en las parcelas de muestreo como en la zona aledaña en el sitio del Remolino (tabla 1). En las parcelas fueron reportadas 17 especies, mientras que en las zonas aledañas solo cinco. Únicamente dos especies epífitas fueron observadas en el muestreo, el resto fueron rupícolas y en mayor medida, terrestres. Las familias más representativas fueron Pteridaceae (cinco especies), Polypodiaceae (cuatro), Thelypteridaceae (tres) y Selaginellaceae (tres). La intensidad del muestreo implementado fue satisfactoria de acuerdo con el valor del estimador ACE, que indica que la riqueza observada representa el $79.3 \%$ de la riqueza esperada.

De acuerdo con el índice de Shannon-Wiener, la mayor diversidad se registró en el hábitat ribereño con un índice que sobrepasa los valores de 1, con un total de 10 especies, destacándose que la mayoría de las especies son terrestres o rupícolas. El sitio de cultivos mostró valores de 0 por parcela, el cual es nulo para calcular diversidad florística ya que sólo se registra una especie en este hábitat. El análisis de varianza corrobora estadísticamente que existen diferencias significativas en la diversidad registrada entre hábitats $(\mathrm{F}=35.999 ; P<0.005)$; la prueba de Tukey mostró que el hábitat ribereño es distinto a los otros dos sitios $(\mathrm{P} \leq 0.005$ en la comparación del hábitat ribereño con respecto a la selva y cultivos).

Diversidad beta. El índice de diversidad beta de Whittaker mostró un valor promedio de $\beta=$ 0.687 entre hábitat ribereño y fragmento de selva. En el análisis de agrupamiento (fig. 2) se observan tres ramas, las primeras están conformadas por las parcelas del hábitat ribereño y fragmento de selva, los cuales presentan una mayor similitud de helechos y licopodios; la tercera está representada por las parcelas del cultivo de cítricos donde se observa una fuerte disimilitud.

El análisis IndVal separó únicamente dos especies (de las 17 registradas en las parcelas) que tuvieron valores de ocurrencia altos en las parcelas de muestreo (valor de indicación alto). Tal es el caso de Pteris grandifolia asociada al hábitat ribereño y Selaginella stellata asociada a la zona de cultivos. 
Tabla 1. Especies registradas dentro de las parcelas de muestreo y zonas aledañas del Remolino. Ambiente: $\mathrm{SE}=$ selva; $\mathrm{AGU}=$ aguachal; $\mathrm{RIB}=$ ribereño; $\mathrm{ANT}=$ antropizada; $\mathrm{CUL}=$ cultivo. Hábito: $\mathrm{R}=$ rupícola; $\mathrm{T}=$ terrestre; $\mathrm{E}=$ epífita.

* No se pudieron identificar a nivel de especie debido a que no se cuenta con material fértil.

\begin{tabular}{|c|c|c|c|}
\hline Familia & Nombre científico & Ambiente & Hábito \\
\hline Aspleniaceae & Asplenium cristatum Lam. & SE & $\mathrm{R}$ \\
\hline \multirow{2}{*}{ Davalliaceae } & Nephrolepis biserrata (Sw.) Schott & $\mathrm{AGU}$ & $\mathrm{T}$ \\
\hline & Nephrolepis hirsutula (G. Forst.) C. Presl & AGU & $\mathrm{T}$ \\
\hline Lonchitidaceae & Lonchitis hirsuta $\mathrm{L}$. & RIB & $\mathrm{T} / \mathrm{R}$ \\
\hline Lygodiaceae & Lygodium venustum $\mathrm{Sw}$ & RIB & $\mathrm{T}$ \\
\hline \multirow[t]{4}{*}{ Polypodiaceae } & Campyloneurum phyllitidis (L.) C.Presl & $\mathrm{SE}$ & $\mathrm{R}$ \\
\hline & Microgramma nitida (J. Sm.) A.R. Sm. & ANT & $\mathrm{E}$ \\
\hline & Pecluma dispersa (A.M. Evans) M.G. Price & SE & $\mathrm{T}$ \\
\hline & $\begin{array}{l}\text { Pleopeltis polypodioides (L.) E.G. Andrews \& } \\
\text { Windham }\end{array}$ & SE & $\mathrm{R}$ \\
\hline \multirow[t]{5}{*}{ Pteridaceae } & Adiantum feei T. Moore ex Fée & $\mathrm{SE}$ & $\mathrm{T} / \mathrm{R}$ \\
\hline & Adiantum tenerum $\mathrm{Sw}$. & $\mathrm{RIB} / \mathrm{SE}$ & $\mathrm{T} / \mathrm{R}$ \\
\hline & $\begin{array}{l}\text { Myriopteris microphylla (Sw.) Grusz \& } \\
\text { Windham }\end{array}$ & SE & $\mathrm{R}$ \\
\hline & Pityrogramma trifoliata (L.) R.M. Tryon & $\mathrm{AGU}$ & $\mathrm{T}$ \\
\hline & Pteris grandifolia $\mathrm{L}$. & RIB & $\mathrm{T}$ \\
\hline Schizaeaceae & Anemia adiantifolia (L.) Swartz & $\mathrm{RIB} / \mathrm{SE}$ & $\mathrm{T}$ \\
\hline \multirow[t]{3}{*}{ Selaginellaceae } & Selaginella hoffmannii Hieron. & RIB, ANT & $\mathrm{T}$ \\
\hline & Selaginella martensii Spring & RIB & $\mathrm{T}$ \\
\hline & Selaginella stellata Spring & CUL & $\mathrm{T}$ \\
\hline Tectariaceae & Tectaria heracleifolia (Wild.) Underw & $\mathrm{SE}$ & $\mathrm{T} / \mathrm{R}$ \\
\hline \multirow[t]{3}{*}{ Thelypteridaceae } & $\begin{array}{l}\text { Christella ovata var. lindheimeri (C.Chr) comb. } \\
\text { ined. }\end{array}$ & RIB & $\mathrm{T}$ \\
\hline & *Thelypteris sp1 & SE & $\mathrm{T}$ \\
\hline & *Thelypteris sp2 & SE & $\mathrm{T}$ \\
\hline
\end{tabular}

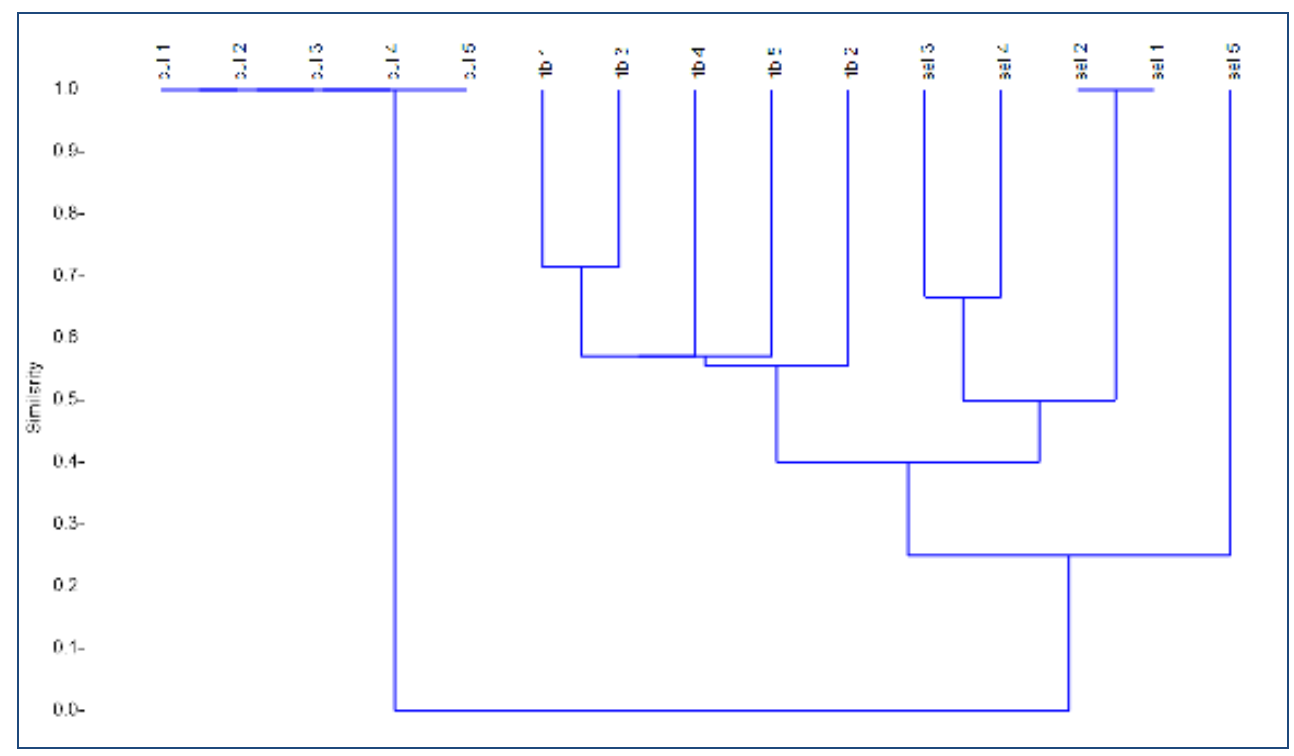


Fig. 2. Dendrograma de similitud basado en la diversidad registrada por parcela. DISCUSIÓN

La riqueza total de especies fue de 22 , lo cual representa en términos generales un número similar de especies en comparación con las registradas en otras selvas de baja altitud presente en la zona centro-sur de Veracruz, así como en Tabasco (Carvajal-Hernández et al., 2018; Cetzal-Ix et al., 2013). Carvajal-Hernández y Krömer (2015) reportan en la selva mediana subcaducifolia en la región central del estado un total de 28 especies que van de los $500 \mathrm{~m}$ hasta el nivel del mar. Por otro lado, el trabajo de Acebey y colaboradores (2015) registran en los Tuxtlas (Veracruz), 115 especies en la selva alta perennifolia en un rango altitudinal que va desde los 0-700 m, sin embargo, separando las especies únicamente de la selva mediana perennifolia, se reportan 23 taxones en una altitud de 650-1 000 m s.n.m. En el estudio de Cetzal-Ix y colaboradores (2013) en un fragmento de selva mediana subperennifolia en Tabasco, fueron observadas 43 especies en Tacotalpa, en altitudes de entre 50 y 630 m s.n.m.; esta alta riqueza de especies se debe a que el muestreo se realizó en una superficie mucho mayor a la del presente estudio, por lo que no es casualidad registrar una riqueza mayor de helechos y licopodios.

El tamaño del área influye en la diversidad de especies (Karger et al., 2011) y en el caso del Remolino, se trata de un fragmento de selva aislado, rodeado de terrenos agrícolas y ganaderos, mientras que los estudios citados en el párrafo anterior corresponden a fragmentos de mayor tamaño con intervalos de altitud más amplios. Por dicha razón, a pesar de que las especies registradas en el Remolino son menos, comparadas con otros estudios, el área podría ser un reservorio o refugio de especies de helechos y licopodios importante, a pesar de las fuertes presiones antropogénicas a las que son sometidos los diferentes hábitats de la zona. Así mismo, otro factor a destacar es que la zona de estudio se encuentra en una latitud mayor comparada con los otros estudios que fueron realizados localidades a latitudes menores, por lo que tal vez la disminución de la riqueza de especies podría estar asociada a restricciones en las condiciones ambientales en latitudes mayores (Moran, 2008). Para corroborar lo anterior será necesario realizar comparaciones con otras selvas tropicales localizadas a mayor latitud, sin embargo, hasta ahora la información al respecto es escasa.

Por otro lado, el hecho de que la zona ribereña contenga la mayor diversidad de especies concuerda con los resultados obtenidos por Kessler (2001) en Bolivia, Kluge y Kessler (2011) en Costa Rica y Carvajal-Hernández y Krömer (2015) en el centro de Veracruz. En dichos trabajos encontraron que la riqueza de especies fue más alta en los márgenes de ríos, lo que confirma la preferencia de los helechos por microambientes dinámicos y complejos con humedad ambiental alta (Vázquez et al., 2015).

La mayor concentración de diversidad de helechos epífitos se encuentra en zonas de media montaña (1 000-2 000 m s.n.m.) con climas templados disminuyendo considerablemente en las zonas de menor altitud, donde la precipitación y la humedad ambiental son menores, lo cual influye en la riqueza de especies de helechos en general y particularmente las especies epífitas (Carvajal-Hernández \& Krömer, 2015; Kreft et al., 2004; Krömer et al., 2005). Esta explicación puede estar relacionada con la escasa representatividad de helechos epífitos en la zona (únicamente dos especies). Los helechos y licopodios tienen un bajo control del potencial evaporativo durante la mayor parte de su ciclo de vida, por lo que dependen de la disponibilidad del agua y de la humedad relativa (Hietz, 2010; Page, 2002). Los valores bajos de humedad ambiental reportados para la zona (40\%) (INEGI, 2009), podrían estar relacionados parcialmente con el escaso número de especies de helechos epífitos. Además de ello, la relativa homogeneidad de microambientes y la poca disponibilidad de nichos adecuados para los helechos en las selvas tropicales de baja altitud como la que se encuentra en la zona de estudio, podría ser otra limitante de la ocurrencia de más especies (Kluge \& Kessler, 2011). 
La única especie que se encuentra enlistada en la NOM-059-SEMARNAT-2010 bajo la categoría de amenazada fue Campyloneurum phyllitidis (L.) C. Presl. Por otro lado, la presencia de Adiantum feei T. Moore ex Fée a $135 \mathrm{~m}$ snm es destacable, debido a que significa un aumento considerable en la amplitud de su intervalo de distribución altitudinal en México, registrado en altitudes mayores a 1300 m s.n.m. Mickel y Smith (2004). Este hallazgo confirma la necesidad de continuar los estudios florísticos en México y en general en zonas tropicales, pues todavía no se conoce la riqueza y distribución de las especies en su totalidad. Los descubrimientos de especies nuevas para la ciencia o los nuevos reportes de distribución son una muestra de que el conocimiento que se tiene (aún de territorios adecuadamente explorados como Veracruz) no está bien fundamentado y existen todavía regiones por explorar (Magaña \& Villaseñor, 2002; Krömer et al., 2015; Carvajal-Hernández et al., 2018), sumado a la falta especialistas en algunos grupos taxonómicos (Mora et al., 2011).

Diversidad beta. Los valores altos del índice de diversidad beta de Whittaker, indican un fuerte recambio de especies entre los hábitats comparados. Los hábitats que tienen una composición de especies de helechos y licopodios similares son la selva y la vegetación ribereña ( $22 \%$ de especies compartidas), ya que la estructura de la vegetación y la disponibilidad de microambientes es semejante. Por otro lado, la composición de especies en el cultivo de cítricos es diferente, debido a que sólo se encontró una especie, el manejo del cultivo (deshierbe), las condiciones de alta radiación solar debido a la falta de dosel y las fuertes temperaturas impiden el establecimiento de más especies de los helechos y licopodios (Carvajal-Hernández \& Krömer, 2015). La tasa de recambio de especies en las zonas de baja altitud es elevada (similar a las zonas de media montaña, aunque con base en un menor número de especies) porque los cambios en la temperatura y/o humedad, naturales o provocados por el disturbio antropogénico son abruptos (Carvajal-Hernández et al., 2017).

La mayor diversidad de especies en el hábitat ribereño puede estar relacionada con la humedad relativa más elevada y suelos más ricos como consecuencia de la disposición de materia producida por la erosión de las laderas (Vázquez et al., 2015). En cambio, en las parcelas de la selva se observa la presencia de un sustrato de roca caliza lo cual provoca escases de agua derivada del escurrimiento elevado ocasionando una nula capacidad de absorción del sustrato (Basáñez et al., 2008; Ticante, 2015). Este resultado podría explicar en parte la alta diversidad beta presente en la zona, ya que a pesar de ser hábitats contiguos y aunque existe un aparente flujo de esporas de un hábitat a otro, éstas no encuentran las condiciones necesarias para prosperar en cualquier ambiente, lo que limita la dispersión de especies.

La presencia de microhábitats en los ecosistemas puede favorecer o limitar la presencia de especies, como ejemplo de ello, en la parcela número cinco de la selva, se obtuvo una mayor diversidad. Esto tiene como explicación que se trató de una parcela con la presencia de una gran pared de roca, propiciando un microhábitat que funciona como refugio de especies ( $\mathrm{p}$ ej. Asplenium cristatum Brack., Campyloneurum phyllitidis (L.) C. Presl, Pecluma dispersa (A.M. Evans) M.G. Price y Myriopteris microphylla (Sw.) Grusz \& Windham). Dichas condiciones microambientales, permiten el establecimiento de las especies de helechos, ya que conservan mayor humedad y una temperatura estable, además de que favorecen la acumulación de materia orgánica (Acebey et al., 2003; Jones et al., 2011).

Especies indicadoras. El análisis IndVal coloca a Pteris grandifolia L. como una especie asociada al hábitat ribereño. Su crecimiento es favorecido en ambientes secundarios desarrollándose en bordes de las selvas, a lo largo de caminos, taludes y vegetación secundaria (Cetzal-Ix et al., 2013; Tejero-Díez, 2007). Por lo tanto, la asociación casi exclusiva a la vegetación ribereña sugiere que puede sobrevivir bajo diferentes condiciones de humedad, temperatura y radiación solar. Por otro lado, en un ambiente perturbado como el cultivo de cítricos, sobresale la presencia de Selaginella stellata Spring, común en áreas desmontadas y bordes de caminos (Mickel \& Smith, 2004), por lo que puede ser considerada como indicadora de ambientes perturbados. 


\section{CONCLUSIONES}

Los resultados indican que el recambio de especies de helechos en tres gradientes ambientales en la zona de estudio fue alto. A pesar de que el área privada de conservación Talhpan es un fragmento de selva rodeado de terrenos ganaderos y de cultivo, tiene un papel importante como refugio de la diversidad de helechos y licopodios en la zona norte del estado de Veracruz. El ensamble de especies de helechos y licopodios en la selva fue distinto al de la vegetación ribereña. Dicha situación, relacionada con la heterogeneidad ambiental, podría tener repercusiones para la conservación de la biodiversidad, ya que la protección del conjunto de microambientes puede asegurar la permanencia de las especies. Además, la ampliación en el rango altitudinal de Adiantum feei corrobora la importancia de continuar con los trabajos florísticos en sitios poco explorados como es el caso de la zona norte de Veracruz. Un punto importante a destacar es el valor cultural del sitio, ya que es un área protegida por una comunidad indígena, para la cual la conservación de su patrimonio natural resulta una tarea primordial.

\section{LITERATURA CITADA}

Acebey, A., Krömer, T., Vázquez-Torres, M., \& Tejero-Díez, J. D. (2015). Helechos y licopodios de la Reserva de la Biosfera Los Tuxtlas, Veracruz, México. Botanical Sciences, 93(2), 1-32.

Acebey, A., Krömer, T., \& Gradstein, S. R. (2003). Species richness and habitat diversification of bryophytes in submontane rain forest and fallows of Bolivia. Journal of Tropical Ecology, 19, 9-18.

Barradas P. M. L., \& Prado, J. (2005). Effects of forest fragmentation on pteridophyte diversity in a tropical rain forest in Brazil. Plant Ecology, 180(1), 87-104. https://doi.org/10.1007/s11258-005-3025-x

Basáñez, A. J., Alanís, J., \& Badillo, E. (2008). Composición florística y estructura arbórea de la selva mediana subperenifolia del ejido "El Remolino", Papantla, Veracrz. Avances En Investigación Agropecuaria, 12(2), 3-21.

Carvajal-Hernández, C. I., \& Krömer, T. (2015). Riqueza y distribución de helechos y licófitos en el gradiente altitudinal del cofre de perote, centro de veracruz, México. Botanical Sciences, 93(3), 601-614. https://doi.org/10.17129/botsci.165

Carvajal-Hernández, C. I., Krömer, T., López-Acosta, J. C., Gómez-Díaz, J. A., \& Kessler, M. (2017). Conservation value of disturbed and secondary forests for ferns and lycophytes along an elevational gradient in Mexico. Applied Vegetation Science, 20(4), 662-672. https://doi.org/10.1111/avsc.12318

Carvajal-Hernández, C. I., Krömer, T., \& Vázquez-Torres, M. (2014). Riqueza y composición florística de pteridobiontes en bosque mesófilo de montaña y ambientes asociados en el centro de Veracruz, México. Revista Mexicana de Biodiversidad, 85(2), 491-501. https://doi.org/10.7550/rmb.41292

Carvajal-Hernández, C. I., Silva-Mijangos, L., Kessler, M., \& Lehnert, M. (2018). Adiciones a la pteridoflora de Tabasco, México: la importancia del bosque mesófilo de montaña. Acta Botanica Mexicana, 124. https://doi.org/10.21829/abm124.2018.1300

Carvajal-Hernández, C. I., Guzmán-Jacob, V., Smith, A. R., \& Krömer, T. (2018). A new species, new combinations in Pecluma and Pleopeltis, and new records for the state of Veracruz. American Fern Journal, 108(4).

Castillo-Campos, G. S., Avendaño-Reyes, S., \& Medina-Abreo, M. E. (2011). Flora y vegetación. In A. Cruz-Angón (Ed.), La biodiversidad en Veracruz: Estudio de estado (pp. 163-179). México, D.F.: Comisión Nacional para el Conocimiento y Uso de la Biodiversidad, Gobierno del Estado de Veracruz, Universidad Veracruzana, Instituto de Ecología, A.C.

Cetzal-Ix, W., Noguera-Savelli, E., \& Martínez-Icó, M. (2013). Diversidad de helechos y licófitos en fragmentos de selva mediana subperennifolia del sur de Tabasco, México. 
Botanical Sciences, 91(3), 261-271.

Chao, A., Chazdon, R. L., Colwell, R. K., \& Shen, T. (2005). Un nuevo método estadístico para la evaluación de la similitud en la composición de especies con datos de incidencia y abundancia. In G. Halfter, J. Soberón, P. Koleff \& A. Melic (Eds) Sobre Diversidad Biológica: el Significado de las Diversidades Alfa, Beta y Gamma (p. 8596). Zaragoza CONABIO-SEA-Grupo Diversitas-CONACYT.

Colwell, R. K. (2013). EstimateS: Statistical estimation of species richness and shared species from samples. Retrieved from Tomado de http://viceroy.eeb.uconn.edu/estimates/.

Dufrene, M., \& Legendre, P. C. (1997). Species Assemblages and Indicator Species: The Need for a Flexible Asymmetrical Approach. Ecological Monographs, 67: 345-366.

Gómez-Pompa, A., Krömer, T., \& Castro-Cortés, R. (Eds.). (2010). Atlas de la flora de Veracruz: Un patrimonio natural en peligro. Xalapa, Veracruz: Comisión del Estado de Veracruz para la conmemoración de la Independencia Nacional y la Revolución Mexicana.

Hammer, Ø., Harper D.A.T. \& Ryan P.D. (2001). Past: Paleontological statistics software package for education and data analysis. Paleontología Electrónica, 4(1), 9.

Hietz, P. (2010). Fern adaptations to xeric environments. In K. Mehltreter, R. L. Walker, \& J. M. Sharpe (Eds.), Fern Ecology (pp. 140-176). New York: Cambridge University Press.

INEGI. (2009). Datos geográficos. Retrieved August 28, 2015, from http://www3.inegi.org.mx/sistemas/mexicocifras/datosgeograficos/30/30124.pdf

Jones, M. M., Szyska, B., \& Kessler, M. (2011). Microhabitat partitioning promotes plant diversity in a mid-elevation tropical montane forest. Global Ecology and Biogeography, 20, 558-569.

Karger, D. N., Kluge, J., Krömer, T., Hemp, A., Lehnert, M., \& Kessler, M. (2011). The effect of area on local and regional elevational patterns of species richness. Journal of Biogeography, 38(6), 1177-1185. https://doi.org/10.1111/j.1365-2699.2010.02468.x

Kelly, D. L., Tanner, E., Lughadha, E. M., \& Kapos, V. (1994). Floristics and biogeography of a rain forest in the Venezuelan Andes. Journal of Biogeography, 21, 421-440.

Kessler, M. (2001). Patterns of diversity and range size of selected plant groups along an elevational transects in the Bolivian Andes. Biodiversity and Conservation, 10, 18971921. https://doi.org/10.1023/A:1013130902993

Kessler, M. (2010). Biogeography of ferns. In K. Mehltreter, L. R. Walker, \& Sharpe J.M. (Eds.), Fern ecology (pp. 22-60). New York: Cambridge University Press.

Kessler, M., \& Bach, H. (1999). Using indicator groups for vegetation classification in speciesrich Neotropical forests. Phytocoenologia, 29(4), 485-502.

Kluge, J., \& Kessler, M. (2011). Influence of niche characteristics and forest type on fern species richness, abundance and plant size along an elevational gradient in Costa Rica. Plant Ecology, 212(7), 1109-1121. https://doi.org/10.1007/s11258-010-9891-x

Koleff, P., Gaston, K. J., \& Lennon, J. J. (2003). Measuring beta diversity for presence absence data. Journal of Animal Ecology, 72, 367-382. https://doi.org/10.1046/j.13652656.2003.00710.x

Kreft, H., Köster, N., Küper, W., Nieder, J., \& Barthlott, W. (2004). Diversity and biogeography of vascular epiphytes in Western Amazonia, Yasuní, Ecuador. Journal of Biogeography, 1463-1476.

Krömer, T., Carvajal-Hernández, C. I., Acebey, A. R., \& Smith, A. R. (2015). A decade of new pteridophyte records for the State of Veracruz, Mexico. American Fern, 105(3), 162175. https://doi.org/10.1640/0002-8444-105.3.162

Krömer, T., Kessler, M., Gradstein, S. R., \& Acebey, A. R. (2005). Diversity patterns of vascular epiphytes along an elevational gradient in the Andes. Journal of Biogeography, 32, 1799-1809.

Magaña, P., \& Villaseñor, J. L. (2002). La flora de México ¡Se podrá conocer completamente? Ciencias, 66, 24-26.

Mickel, J. T., \& Smith, A. R. (2004). The pteridophytes of Mexico. Memoirs of the New York Botanical Garden, 88, 1-1054. 
Recibido: 18/diciembre/2017

Aceptado: 8/octubre/2018
Mora, C., Tittensor, D. P., Adl, S., Simpson, G. A., \& Worm, B. (2011). How many species are there on earth and in the ocean? PLoS Biology, 9(8).

Moran, R. (2008). Diversity, biogeography and floristics. In T. Ranker \& C. Haufler (Eds.), Biology and Evolution of Ferns and Lycophytes (pp. 367-394). U.K.: Cambridge Press.

Moreno, C. E. (2001). Métodos para medir la biodiversidad. Zaragoza, España: Cooperación Iberoamericana (CYT ED), Unesco (Orcyt) y SEA.

Page, C. (2002). Ecological Strategies in Fern Evolution; a neoteridological overview. Reveiw of Palaeobotany \& Palynology, 119, 1-33.

PPGI (Pteridophyte Phylogeny Group I). (2016). A community-derived classification for extant lycophytes and ferns. Journal of Systematics and Evolution, 54(6), 563-603. https://doi.org/10.1111/jse.12229

Salazar, L., Homeier, J., Kessler, M., Abrahamczyk, S., Lehnert, M., Krömer, T., \& Kluge, J. (2015). Diversity patterns of ferns along elevational gradients in Andean tropical forests. Plant Ecology and Diversity, 8(1), 13-24. https://doi.org/10.1080/ 17550874.2013.843036

Sarukhán, J., Soberón, J., \& Larson-Guerra, J. (1996). Biological Conservations in a High Betadiversity Country. In T. Di Castri, F.; Younés (Ed.), Biodiversity Science and Development: Towards a New Partnership (pp. 246-263). Paris, France: CAB International-IUBS.

SEMARNAT. (2009). Biodiversidad. Retrieved from http://www.semarnat.gob.mx/ informacionambiental/Documents/pdf/cap_ 4 biodiversida

StatSoft Inc. (2007). Statistica (data analysis software system) version 8.0. Retrieved from www.statsoft.com

Tejeda-Cruz, C., Mehltreter, K., \& Sosa, V. (2008). Indicadores ecológicos multitaxonomicos. In R. H. Manson, V. Hernández-Ortíz, S. Gallina, \& K. Mehltreter (Eds.), Agroecosistemas cafetaleros de Veracruz: Biodiversidad, manejo y conservación (pp. 271-278). México, D.F.

Tejero-Díez, J. D. (2007). La riqueza florística del estado de México: licopodios y helechos. Adumbrationes Ad Summae Editionem, 27, 1-32.

Tejero-Díez, J. D., Torres-Díaz, A., Mickel, J. T., Mehltreter, K., \& Krömer, T. (2011). Helechos y licopodios. In M. E. Medina-Abreo (Ed.), La Biodiversidad en Veracruz: Estudio de Estado. (pp. 97-115). México, D.F.: Comisión Nacional para el Conocimiento y Uso de la Biodiversidad, Gobierno del Estado de Veracruz, Universidad Veracruzana, Instituto de Ecología, A.C.

Ticante, D. (2015). Estructura forestal y uso etnobotánico en el área privada de conservación "Talhpan", Papantla, Veracruz, México. Universidad Veracruzana.

Ulloa, U. E., Acevedo-Rodríguez, P., Beck, S., Belgrano, M. J., ..., \& Jørgensen, P. M. (2017). An integrates assessment of the vascular plant species of the Americas. Science, 358, 1614-1617.

Vázquez-Torres, M., Carvajal-Hernández, C. I., \& Aquino-Zapata, A. M. (2010). Áreas Naturales Protegidas. In G. Benitez-Badillo \& C. Welsh (Eds.), Atlas del Patrimonio Natural, Histórico y Cultural de Veracruz. Tomo 1 (pp. 247-274). Xalapa, Veracruz: Comisión del Estado de Veracruz para la conmemoración de la Independencia Nacional y la Revolución Mexicana.

Vázquez, G., García-Franco, J. G., Castillo, G., Escobar, F., Guillén, A., Martínez, M. L., ... Galindo, J. (2015). Ecosistemas ribereños: un paisaje fragmentado. Biodiversitas, 119, 7-11.

Villaseñor, J. L. (2016). Checklist of the native vascular plants of Mexico. Revista Mexicana de Biodiversidad, 87(3), 559- 902. 